\title{
Comportamiento mecánico y estudio in situ de la evolución del daño en el grafito y en un compuesto $\mathrm{C} / \mathrm{C}$ a 77 y a $300 \mathrm{~K}$
}

\author{
A. SALAZAR, J. Y. PASTOR, J. LLORCA \\ Departamento de Ciencia de Materiales. Universidad Politécnica de Madrid. E.T.S.I. de Caminos. \\ Ciudad Universitaria, E-28040-Madrid.
}

\begin{abstract}
En este trabajo se han evaluado las propiedades mecánicas a 77 y a $300 \mathrm{~K}$ de dos grafitos policristalinos con microestructuras muy diferentes y de un compuesto $\mathrm{C} / \mathrm{C}$. Se realizaron ensayos de flexión en tres puntos y de tenacidad, y en éstos últimos se midió in situ la iniciación y la evolución del daño en el fondo de la entalla con un microscopio de larga distancia focal. Los resultados obtenidos muestran que las temperaturas criogénicas no afectan el comportamiento mecánico de los compuestos de carbono estudiados. Además, la porosidad presente en los grafitos es el mecanismo de rotura dominante mientras que en el material compuesto, la intercara débil entre matriz y haz de fibras detiene y desvía las grietas nucleadas en la matriz, presentando el mejor comportamiento mecánico.
\end{abstract}

Palabras clave: grafito, material compuesto C/C, micromecanismos de rotura, criogenía, propiedades mecánicas.

\section{Mechanical behavior and in situ study of the damage evolution at 77 and at $300 \mathrm{~K}$ of graphite and $\mathrm{C} / \mathrm{C}$ composites.}

The mechanical properties at $77 \mathrm{~K}$ and at $300 \mathrm{~K}$ were determined in two polycristalline graphites with different microstructures and in a $\mathrm{C} / \mathrm{C}$ composite. The flexure strength and the fracture toughness were evaluated and the micromechanic failure was studied in situ with a long distance microscope. The results obtained showed that the mechanical properties of these carbon materials are not affected by the cryogenic temperatures. The porosity is the main responsible of the mechanical failure in the graphites while in the composite, the matrix/yarn interface deflect the crack nucleated in the mattrix, presenting the best mechanical behavior.

Keywords: Graphite, C/C composite, fracture micromechanisms, cryogenic, mechanical properties.

\section{INTRODUCCIÓN}

El grafito y los compuestos de carbono se caracterizan por poseer excelente resistencia a la corrosión, estabilidad mecánica y estructural a alta temperatura, elevada conductividad térmica y eléctrica, facilidad de mecanizado y precio razonable. Todo ello hace que estos materiales tengan un papel preponderante en las aplicaciones a alta temperatura de la industria electrometalúrgica y química. Pero estas propiedades también son relevantes en sus posibles aplicaciones criogénicas, no existiendo información en la literatura acerca de las propiedades criogénicas de estos materiales. Por tanto, uno de los objetivos de este estudio será la determinación de la resistencia a flexión y de la tenacidad de fractura, a 77 y $300 \mathrm{~K}$, de dos grafitos isótropos policristalinos con microestructuras muy diferentes y de un material compuesto C/C. Esto permitirá analizar la evolución del comportamiento mecánico en función de la temperatura y la microestructura del material.

La evolución del daño mediante la observación in situ de la nucleación y crecimiento de grietas en función de la carga aplicada en los materiales compuestos de carbono permitirá determinar una conexión entre la microestructura y los mecanismos de rotura responsables de la fractura de los materiales compuestos de carbono. Esto ha sido estudiado por muy pocos investigadores [1], siendo otro de los objetivos de la presente investigación. Para ello se ha desarrollado un dispositivo experimental que consta de un microscopio de larga distancia focal con el que se consigue visualizar la iniciación y evolución del daño alrededor del fondo de una entalla durante los ensayos de tenacidad a $300 \mathrm{~K}$.

\section{MATERIALES}

Se han analizado dos grafitos isótropos (UC28 y EM22), con microestructuras muy distintas y un material compuesto C/C (CF270). La empresa Schunk Ibérica fabricó los tres materiales en forma de placas paralelepípedas con dimensiones: i) $9.5 \mathrm{~mm} \times 50 \mathrm{~mm} \times 120 \mathrm{~mm}$ para los grafitos, y ii) $7 \mathrm{~mm}$ x $40 \mathrm{~mm}$ x $150 \mathrm{~mm}$ para el material compuesto.

El grafito UC28 se obtuvo a partir de partículas de coke, con un tamaño máximo de $800 \mu \mathrm{m}$, y de alquitrán, que actuó como conglomerante. Ambos componentes se mezclaron a $150^{\circ} \mathrm{C}$, se conformaron por extrusión, y se carbonizaron a $800^{\circ} \mathrm{C}$ en vacío durante un mes. El proceso de impregnación con alquitrán y carbonización se repitió varias veces con el fin de aumentar la densidad y mejorar la resitencia a la oxidación. La grafitización se realizó a $2600{ }^{\circ} \mathrm{C}$ durante dos semanas, presentando el material resultante buena resistencia a la oxidación, y una elevada conductividad térmica y eléctrica. Este material se emplea en los pantógrafos de las catenarias del ferrocarril, y en las boquillas para la extrusión de aluminio y vidrio. 
El grafito EM22 se fabricó con las mismas materias primas que el UC28, pero el tamaño de grano máximo de coke fue de $7 \mu \mathrm{m}$. La mezcla de alquitrán y coke se prensó isostáticamente, y se carbonizó en vació a $800{ }^{\circ} \mathrm{C}$ durante un mes. La grafitización se realizó a $2800^{\circ} \mathrm{C}$ durante cuatro semanas. Finalmente se obtuvo un material denso, homogéneo y con alta resistencia al desgaste. Este material se emplea como electrodo en los motores eléctricos de gran potencia.

El material compuesto C/C CF270, se fabricó apilando un tejido bidimensional de haces de unas 500 fibras de carbono impregnado en una resina epoxy fenólica. El conjunto se consolidó aplicando presión, se carbonizó en vacío durante un mes a $800{ }^{\circ} \mathrm{C}$ y se grafitizó durante otro mes a $3000{ }^{\circ} \mathrm{C}$. El material resultante se caracteriza por presentar una gran estabilidad estructural a elevadas temperaturas, siendo empleado como moldes para sintetizar diamante.

\section{TÉCNICAS EXPERIMENTALES}

La densidad y el módulo de elasticidad de los tres materiales se midieron sobre las placas tal y como fueron recibidas. La densidad se determinó a partir de las dimensiones y la masa de las placas, y el módulo de elasticidad dinámico, E, y el de cizalladura, $G$, a partir de las frecuencias de resonancia fundamentales de las placas en modo de flexión y de torsión respectivamente. (Grindosonic MK4i, J. W. Lemmens).

Las propiedades mecánicas se midieron en máquinas servo-hidráulicas (Instron Serie 8500 Plus, UK). Los ensayos de flexión en tres puntos se realizaron sobre probetas prismáticas con un canto de 4 ó $5 \mathrm{~mm}$, 16 ó $20 \mathrm{~mm}$ de distancia entre apoyos y un espesor 2.5 veces el canto. La carga aplicada se midió con una célula de carga de $\pm 1 \mathrm{KN}$, y el desplazamiento del punto de carga con un LVDT de recorrido $\pm 1 \mathrm{~mm}$.

En los ensayos criogénicos, el dispositivo de flexión fue introducido en una vasija de acero inoxidable recubierta de poliuretano. El fondo de la vasija y el punto de aplicación de la carga estaban conectados al pistón hidráulico y a la célula de carga con sendas varillas huecas de acero inoxidable. El acero inoxidable se utilizó por su baja conductividad térmica, limitando el enfriamiento de la célula de carga y del actuador. La probeta se colocó en el dispositivo de ensayo a temperatura ambiente y se sometió a carga constante (unos $5 \mathrm{~N}$ ) mientras se sumergía en nitrógeno líquido $(77 \mathrm{~K})$ y hasta que se terminó el proceso de enfriamiento. Este proceso se consideró terminado cuando el pistón dejó de moverse para compensar las contracciones térmicas. En ese momento se inició el ensayo, ya que se suposo que la condición de equilibrio térmico se había alcanzado en el sistema y la temperatura de la probeta sumergida es la del nitrógeno líquido (77 K).

Todos los ensayos se realizaron manteniendo la velocidad de desplazamiento del pistón constante. La velocidad de desplazamiento del pistón fue de: i) $20 \mu \mathrm{m} / \mathrm{min}$ para los grafitos y de $50 \mu \mathrm{m} / \mathrm{min}$ para el compuesto a temperatura ambiente, ii) las velocidades se duplicaron en los ensayos a $77 \mathrm{~K}$.

En los ensayos de tenacidad se emplearon probetas prismáticas con una profundidad de entalla del $30 \%$ del canto, realizadas con una cortadora de hilo de diamante de $130 \mu \mathrm{m}$ de diámetro. El espesor de las probetas fue igual al de las placas originarias y la relación entre la separación entre apoyos y el canto fue de 3 para los grafitos y de 4.3 para el material compuesto. La velocidad de desplazamiento del pistón fue de $100 \mu \mathrm{m} / \mathrm{min}$ para los grafitos y de $120 \mu \mathrm{m} / \mathrm{min}$ para el material compuesto. Se empleó un microscopio de larga distancia focal (Questar), un monitor de video y una cámara digital de alta resolución (Nikon CoolPix950) para observar in situ la iniciación y evolución del daño alrededor del fondo de la entalla en función de la carga aplicada.

Por último, las superficies de fractura de las probetas ensayadas se analizaron en un microscopio electrónico de barrido (JEOL JSM 6300) para analizar los mecanismos de rotura en cada material.

\section{RESULTADOS Y DISCUSIÓN}

\subsection{Comportamiento mecánico}

En la tabla I se encuentran recogidos los valores de la densidad y de los módulos de elasticidad y cizalladura con sus correspondientes errores cuadráticos medios de los tres materiales a partir de 5 medidas realizadas.

Tabla I. Densidad y Módulos De Elasticidad

\begin{tabular}{|c|c|c|c|}
\hline & $\rho\left(\mathrm{g} / \mathrm{cm}^{3}\right)$ & E $(\mathrm{GPa})$ & $\mathbf{G}(\mathrm{GPa})$ \\
\hline UC28 & $1.60 \pm 0.02$ & $7.7 \pm 0.5$ & $2.7 \pm 0.4$ \\
\hline EM22 & $1.87 \pm 0.02$ & $12.7 \pm 0.4$ & $5.4 \pm 0.3$ \\
\hline CF270 & $1.34 \pm 0.04$ & $17.8 \pm 0.5$ & $6.7 \pm 0.4$ \\
\hline
\end{tabular}

El grafito UC28 presentó una densidad y módulos elásticos más bajos que los del grafito EM22. Como revela la superficie de fractura de las probetas ensayadas a flexión (Fig. 1), poros con un tamaño medio de $100 \mu \mathrm{m}$ predominan en toda la superficie. Su fracción volumétrica es del $30 \%$ y se originaron al quedar atrapados los compuestos volátiles formados durante la etapa de carbonización. La superficie de fractura del grafito EM22 (Fig. 2a) presentó una estructura más compacta con una porosidad del orden del $15 \%$ y un tamaño de poro un orden de magnitud menor que en el grafito UC28. La microestructura en ambos grafitos es isótropa. El material compuesto CF270 se caracterizó por tener la menor densidad, estando los poros concentrados en las intersecciones de los haces de fibras orientados en distintas direcciones (Fig. 3a). Estos poros surgieron durante el proceso de impregnación debido a la dificultad de infiltrar la resina fenólica a través del tejido bidimensional. Además, el compuesto exhibió las mejores constantes elásticas por el efecto reforzante de las fibras.

Los valores de la resistencia a flexión a 77 y a $300 \mathrm{~K}$ determinado mediante la expresión existente para una viga elástica de la teoría de Resistencia de Materiales se presentan en la Tabla II junto con sus errores cuadráticos medios. Estos valores se obtuvieron realizando cinco ensayos para cada material y temperatura.

TABla II. ResistenCIA A FLEXIÓN EN MPa A 300 y A 77 K

\begin{tabular}{|c|c|c|}
\hline & $300 \mathrm{~K}$ & $\mathbf{7 7} \mathrm{K}$ \\
\hline UC28 & $9.3 \pm 0.5$ & $10.1 \pm 0.7$ \\
\hline EM22 & $61 \pm 3$ & $65 \pm 2$ \\
\hline CF270 & $100 \pm 3$ & $105 \pm 2$ \\
\hline
\end{tabular}

Las resistencias a flexión de los dos grafitos resultaron ser muy diferentes. Los grandes poros, muchos de ellos interconectados, en el grafito UC28 actuaron como concentradores de tensiones provocando la aparición de grietas y la posterior fractura para estados de carga muy reducidos. Por el contrario, el grafito EM22 se caracterizó por presentar una superficie de fractura plana en la que se pudo observar la rotura por clivaje de los granos de grafito cristalino rodeados de una matriz dúctil (Fig. 2b). La fractura por clivaje de los cristales Boletín de la Sociedad Española de Cerámica y Vidrio. Vol. 41 Núm. 2 Marzo-Abril 2002 


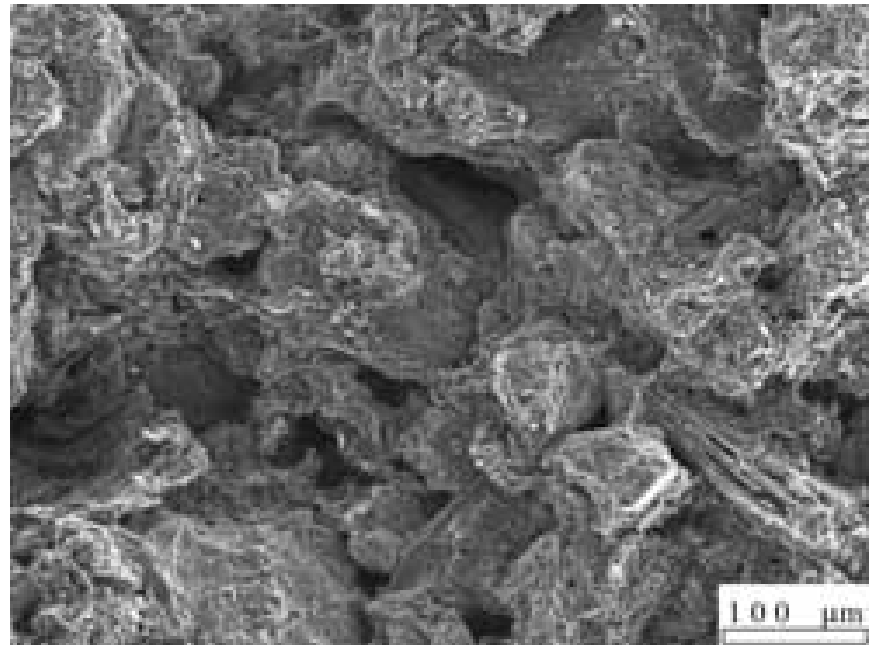

Figura 1. Superficie de fractura del grafito UC28.
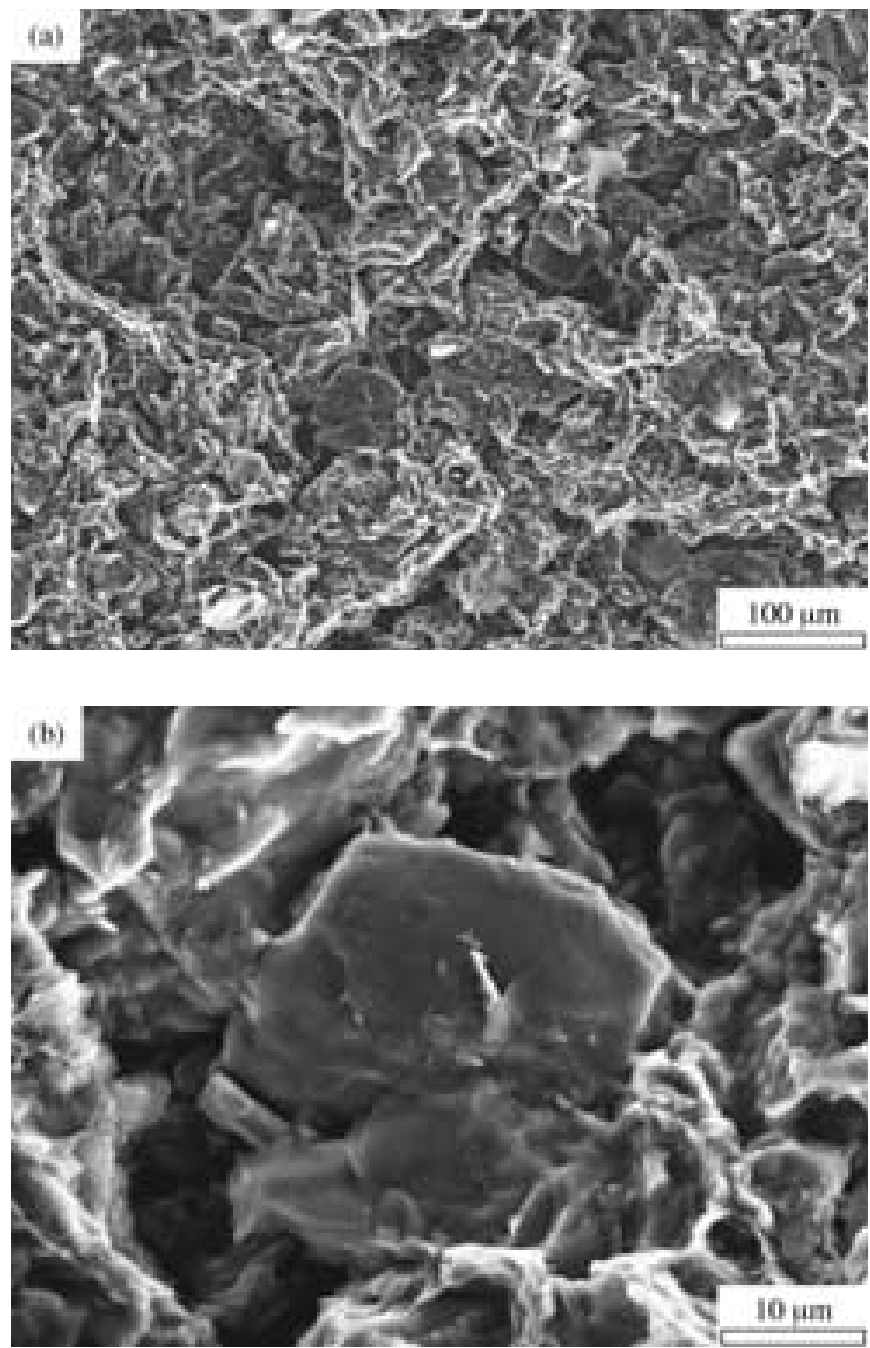

Figura 2. (a) Superficie de fractura del grafito EM22. (b) Detalle en el que se muestra la rotura por clivaje de un grano de grafito.
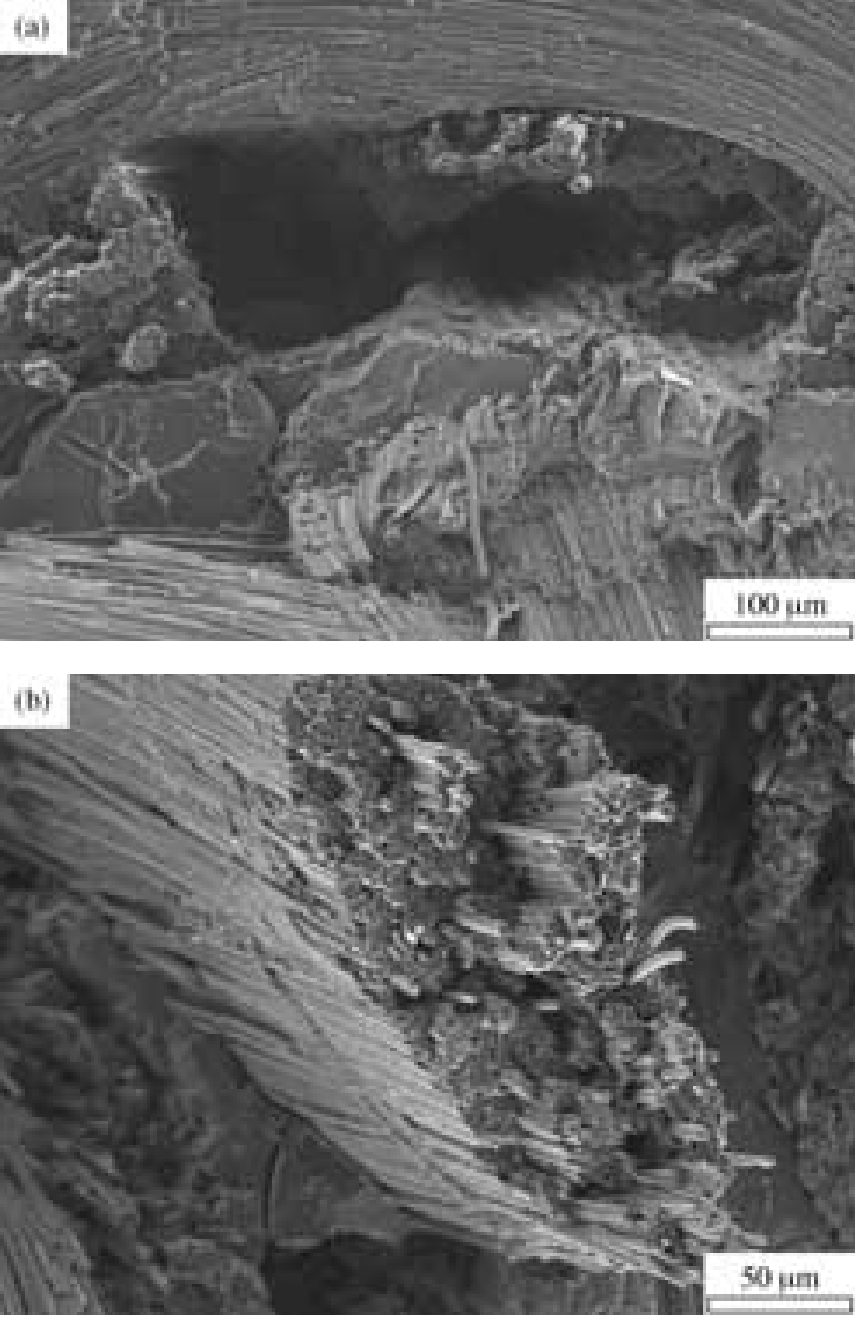

Figura 3. (a) Superficie de fractura del material compuesto CF270. (b) Haz de fibras sobresaliendo de la superficie de fractura de la matriz.

de grafito a lo largo del plano basal se debe a las débiles fuerzas de Van der Waals que unen las capas hexagonales planas de carbono que lo constituyen.

Los valores de la resistencia a flexión obtenidos para los grafitos están de acuerdo con los valores típicos de grafitos isótropos policristalinos [2-3], y las diferencias entre ellos responden a las predicciones del modelo de fractura de Burchell [3]. Según éste, los factores que determinan la resistencia mecánica de estos materiales son el diámetro de los poros, que controla la nucleación de grietas, y el diámetro de las partículas de coke, que facilitan su propagación hasta alcanzar la longitud crítica.

El material compuesto CF270 presentó los mejores valores de la resistencia a flexión debido al efecto reforzante de las fibras. El análisis fractográfico muestra cómo los haces de fibras fueron expulsados del plano de rotura de la matriz (Fig. 3a), debido a la presencia de una intercara débil matriz/haz de fibras, lo que provocó la desviación de las grietas nucleadas en la matriz, evitando la rotura catastrófica del material.

Los valores de la resistencia a flexión a $77 \mathrm{~K}$ de los tres materiales resultaron ser análogos a los obtenidos a temperatura ambiente, no existiendo tampoco diferencias entre las superficies de fractura de los materiales ensayados a ambas temperaturas. 


\subsection{Tenacidad y micromecanismos de fractura}

En la tabla III se muestran los valores de la tenacidad de fractura aparente, con sus correspondientes errores cuadráticos medios, obtenidos tras la realización de cinco ensayos para cada material y temperatura. El cálculo de la magnitud se realizó utilizando la expresión de Guinea et al. [4] a partir de la carga máxima y la longitud inicial de la entalla. Este valor así obtenido es sólo un valor aparente de la tenacidad del material, ya que no se tuvo en cuenta el crecimiento estable de las grietas antes de alcanzarse carga máxima (bien documentado tanto para el grafito [1-5] como para los compuestos C/C [5, 6-7]), ni la influencia del radio del fondo de la entalla $(\approx 90 \mu \mathrm{m})$. Sin embargo, este último factor puede ser obviado cuando la longitud de los defectos presentes en el material son del orden del radio de la entalla tal y como documentó Li et al. [8].

La utilización del microscopio de larga distancia focal permitió visualizar los micromecanismos responsables de rotura y correlacionarlos con el estado tensional sufrido por el material en cada instante. Las figuras 4a 4b y 4c muestran la región próxima al fondo de la entalla en el material UC28, y el estado tensional asociada a cada micrografía se encuentra reflejado en la curva carga-desplazamiento de la Fig. 5. Al comienzo del ensayo, el fondo de la entalla se hallaba libre de defec-
TABLA III: TENACIDAD DE FRACTURA APARENTE EN MPA* ${ }^{1 / 2}$ A $300 \mathrm{~K}$ Y A $77 \mathrm{~K}$

\begin{tabular}{|c|c|c|}
\hline & $\mathbf{3 0 0} \mathbf{~}$ & $\mathbf{7 7} \mathbf{~}$ \\
\hline UC28 & $0.40 \pm 0.02$ & $0.49 \pm 0.01$ \\
\hline EM22 & $1.12 \pm 0.02$ & $1.15 \pm 0.02$ \\
\hline CF270 & $3.0 \pm 0.3$ & $3.1 \pm 0.2$ \\
\hline
\end{tabular}

tos, comenzándose a nuclear microgrietas a su alrededor al alcanzarse el $80 \%$ de la carga máxima. En ese momento, no sólo el fondo de la entalla estaba agrietado sino también la zona circundante (Fig. 4b). Estas grietas debieron nuclearse a partir de poros próximos a la superficie que se encontraban en la región de concentración de tensiones debida a la entalla, emergiendo a la superficie al unirse las grietas generadas en los distintos poros. Como puede apreciarse en la Fig. 4c estas grietas no progresaron.

El mecanismo responsable de la fractura del material EM22 fue análogo al del UC28. Su observación presentó muchas más dificultades puesto que las microfisuras en el fondo de la entalla comenzaron a nuclearse al alcanzarse el 95\% de la carga máxima, teniendo una longitud aproximada de unas $100 \mu \mathrm{m}$. Estas grietas crecieron de forma inestable al llegar a carga máxima rompiendo la probeta en dos trozos. Así pues, los valores más altos de la tenacidad están asociados a una microestructura más fina (Fig. 2a), puesto que se necesitan cargas mayores para nuclear grietas en poros de menor
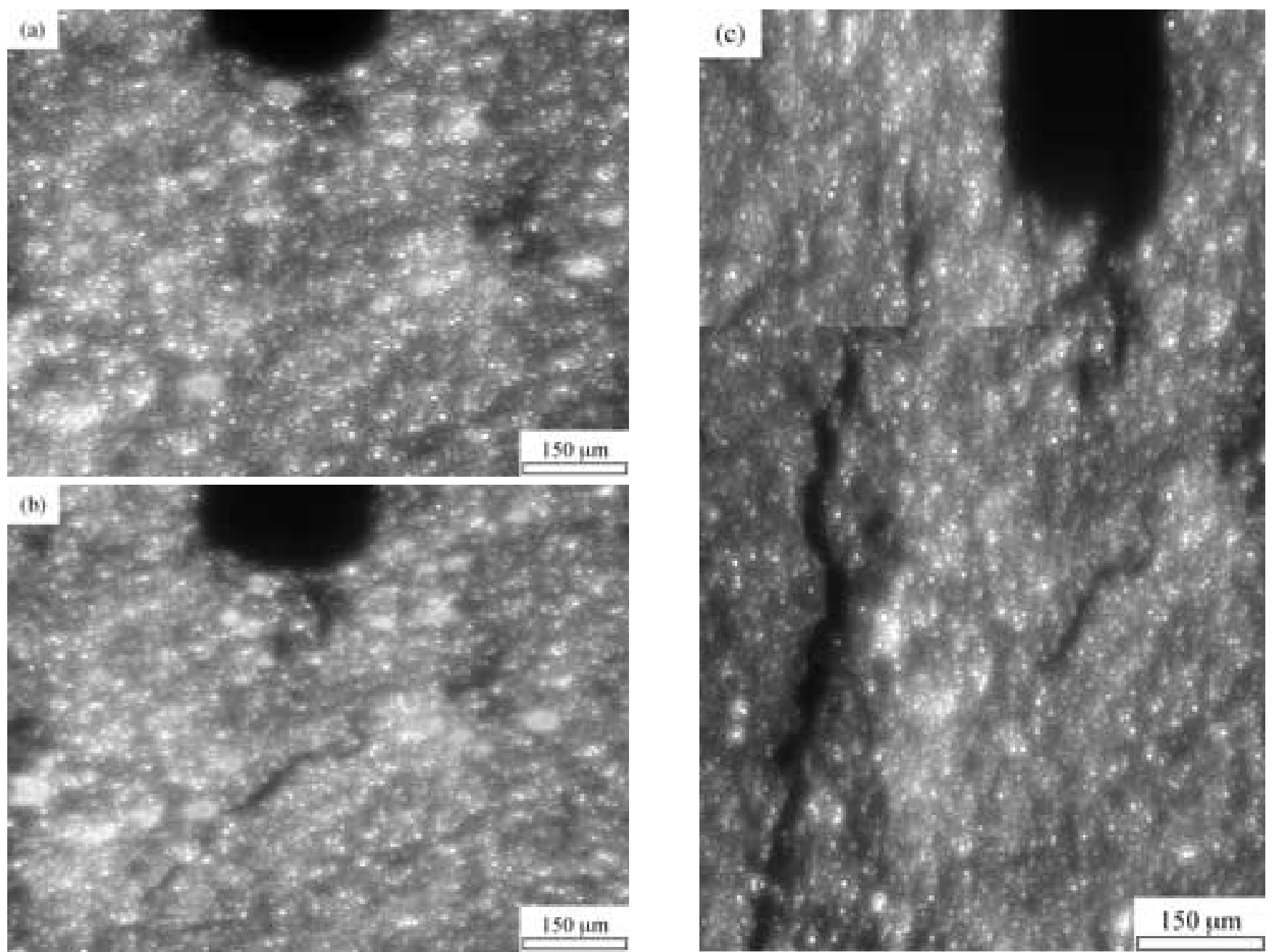

Figura 4. Ensayos de fractura a $300 \mathrm{~K}$ del grafito UC28. (a) Micrografía del fondo de la entalla. (b) Micrografía al alcanzarse la carga máxima. (c) Micrografía correspondiente a la rama de descarga. 


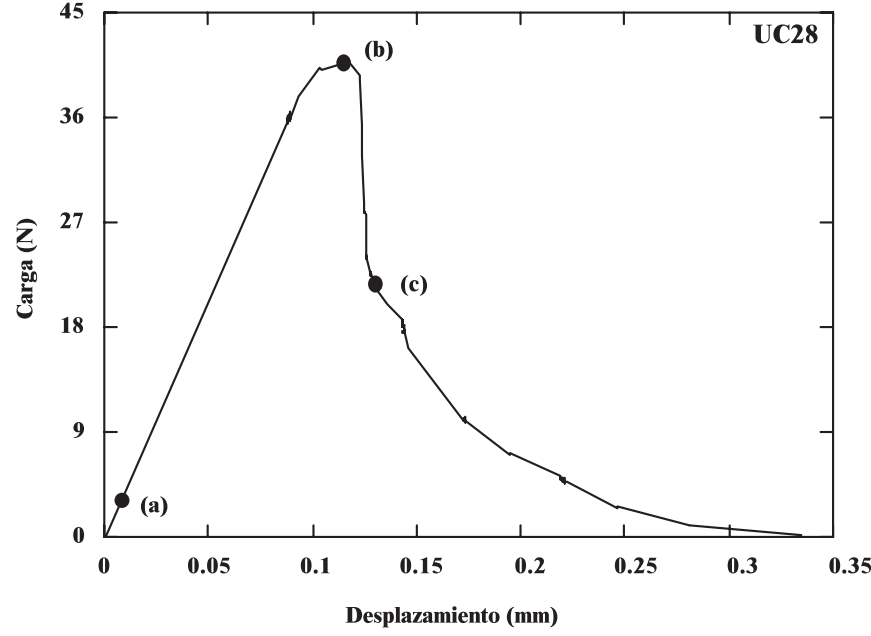

Figura 5. Curva carga-desplazamiento correspondiente al ensayo de tenacidad a $300 \mathrm{~K}$ del grafito UC28 en el que se muestra la situación asociada a las micrografías de la Figura 4. tamaño. Por contraposición, el mecanismo de rotura es más frágil puesto que las grietas, una vez nucleadas, crecieron de manera inestable. Este comportamiento es completamente opuesto al del grafito UC28, en el que la propagación del daño se realizó de una manera estable al producirse la coalescencia de grietas y poros debido a su microestructura más abrupta.

El material CF270 presenta un comportamiento elástico y lineal hasta alcanzar el $70 \%$ de la carga máxima (Fig. 7), momento en el que comienzaron a nuclearse grietas en la matriz alrededor del fondo de la entalla (Fig. 6b). El material se hace más flexible como consecuencia de la propagación de las grietas en la matriz (Fig. 7) y la carga sigue aumentando debido a que son los haces de fibras perpendiculares al plano de la grieta los que soportaron la carga (Fig. 6c). Así pues, los haces de fibras aumentaron la tenacidad del compuesto ya que por un lado, propiciaron la propagación de la fisura a lo largo de la interfase matriz/haz, y por otro, cosieron la fisura detrás de la grieta y dificultaron su propagación (Fig. 6d). Como puede apreciarse en la Fig. 3b, la superficie de fractura del haz es plano, es decir, el aumento de tenacidad fue debida a haces de fibras y no a las fibras individuales dentro del haz, lo que limita este aumento.
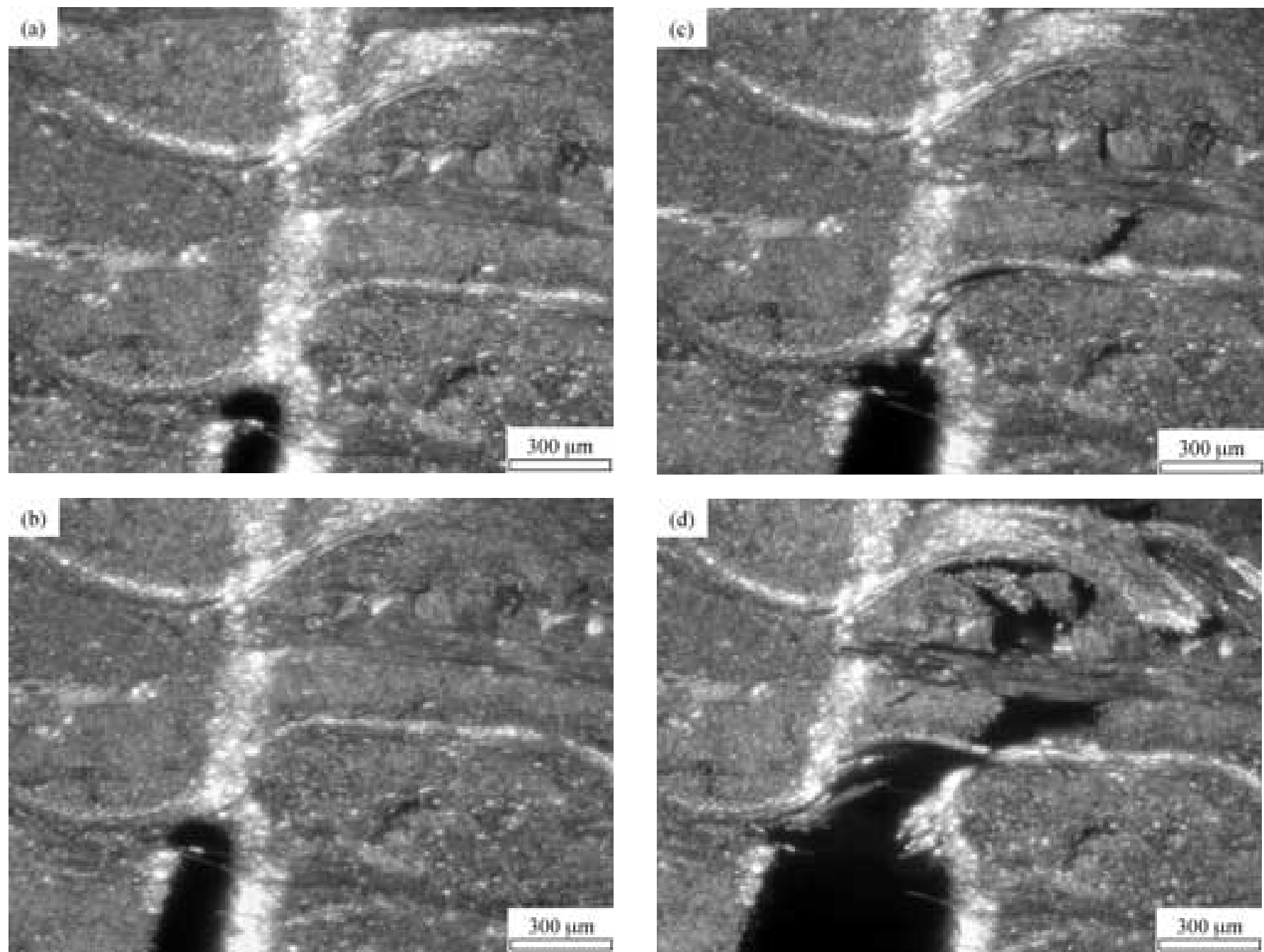

Figura 6. Ensayos de fractura a $300 \mathrm{~K}$ del compuesto CF270. (a) Micrografía del fondo de la entalla en el inicio del ensayo. (b) Micrografía correspondiente al 70\% de la carga máxima. (c) Micrografía asociada a carga máxima. (d) Micrografía en la rama de descarga. 
En los ensayos de tenacidad realizados a $77 \mathrm{~K}$ fue imposible estudiar los micromecanismos de fractura, pero las curvas carga-desplazamiento presentaron un aspecto idéntico a las obtenidas a $300 \mathrm{~K}$, lo que nos permite inferir que el comportamiento fue análogo al observado a temperatura ambiente.

\section{CONCLUSIONES}

Se ha estudiado el comportamiento mecánico a 77 y a 300 $\mathrm{K}$ de dos grafitos policristalinos y de un compuesto C/C, así como la iniciación y evolución del daño in situ alrededor del fondo de la fisura en los ensayos de tenacidad. Los resultados obtenidos muestran que los materiales compuestos de carbono son insensibles al efecto de las temperaturas criogénicas.

El mecanismo responsable del fallo en los grafitos es la presencia de poros. La iniciación y evolución del daño está influenciada por la microestructura existente en el material. Así, en el grafito con una microestructura más basta, caracterizada por defectos microestructurales de gran tamaño, las grietas se nuclean para un estado de carga bajo, en una región amplia alrededor del fondo de la entalla, evolucionando de manera estable mediante la unión de poros y grietas. Por el contrario, en el grafito con microestructura más fina, las grietas se nuclean y concentran en el extremo de la entalla para cargas muy próximas a la de rotura, creciendo de forma inestable, lo que conduce a una rotura catastrófica del material.

En el material compuesto el daño se nuclea en la matriz, siendo los haces de fibras los que impiden un crecimiento inestable de la grieta, cosiéndola a su paso y soportando la carga en el material. Todo esto produce un aumento de la resistencia mecánica, dando lugar a valores muy superiores a los de los grafitos.

\section{AGRADECIMIENTOS}

Los autores agradecen a la compañia SCHUNK IBÉRICA el suministro de los materiales.

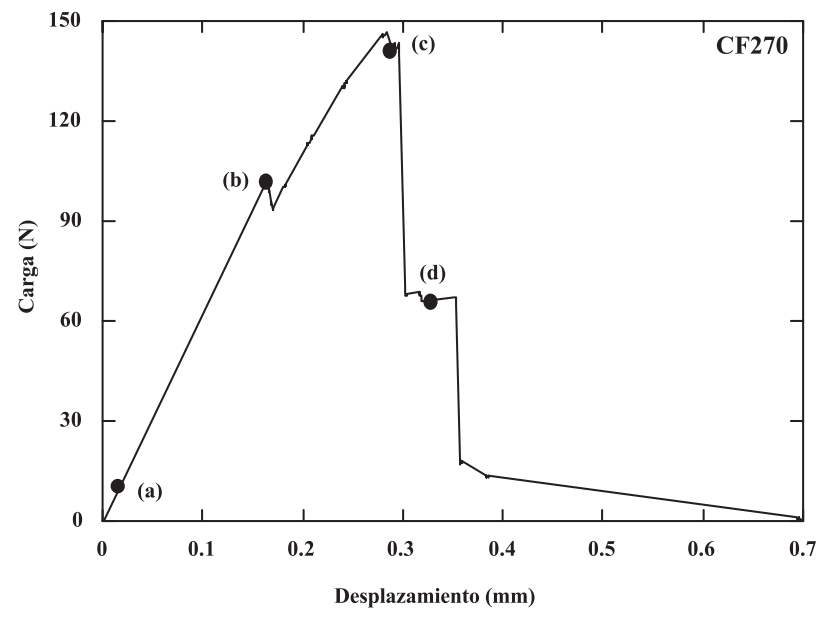

Figura 7. Curva carga-desplazamiento correspondiente al ensayo de tenacidad a $300 \mathrm{~K}$ del compuesto CF270 en el que se muestra la situación asociada a las micrografías de la Figura 6.

\section{BIBLIOGRAFÍA}

1. M. Sakai, J. I. Yoshimura, Y. Goto y M. Inagaki, "R-curve behavior of a polycrystalline graphite: microcracking and grain bridging in the wake region", J. Am. Ceram. Soc. 71, 609 (1988).

2. T. D. Burchell, "A microstructurally based fracture model for polygranular graphites", Carbon 34, 297 (1996).

3. R. Fujiura, T. Kojima, M. Komatsu y I. Mochida, "Mechanical properties of binderless carbon mould prepared from heat-treated mesophase pitch of controlled grain size", Carbon 33, 1061 (1995).

4. G. V. Guinea, J. Y. Pastor, J. Planas y M. Elices, "Stress intensity factor, compliance and CMOD for a general three-point bend specimen", Int. J. Fracture 89, 103 (1998).

5. M. Sakai y H. Kurita, "Size-effect on the fracture toughness and the R-curve of carbon materials", J. Am. Ceram. Soc. 79, 3177 (1996).

6. M. Gomina, D. Themines, J. L. Chermant y F. Ostertock, "An energy evaluation for C/SiC composite materials", Int. J. Fracture 34, 219 (1987).

7. J. Llorca y M. Elices, "Fracture resistance of fiber reinforced ceramic mattrix composites", Acta Metall. Mater. 38, 2485 (1990).

8. M. Li, M. Tsujimura y M. Sakai, "Crack-face grain interlocking/bridging of a polycrystalline graphite: the role in mixed mode fracture", Carbon 37, 1633 (1999).

Recibido: 17.04 .01

Aceptado: 13.11 .01 